\title{
Decaheme Cytochrome MtrF Adsorption and Electron Transfer on Gold Surface
}

\author{
Tao $\mathrm{Wei}^{1{ }^{*}}$ Heng Ma, ${ }^{1}$ Aiichiro Nakano, ${ }^{2,3,4,5}$
}

1. Dan F. Smith Department of Chemical Engineering, Lamar University, Beaumont, Texas, 77710, United States

2. Department of Computer Science, University of Southern California, Los Angeles, California 90089-0781, United States

3. Department of Physics \& Astronomy, University of Southern California, Los Angeles, California 90089-0484, United States

4. Molecular and Computational Biology Section, Department of Biological Sciences, University of Southern California, Los Angeles, California 90089-0371, United States

5. Mork Family Department of Chemical Engineering \& Materials Science, University of Southern California, Los Angeles, California 90089-1211, United States

\section{Corresponding Author}

* To whom all correspondence should be addressed.

Email address: twei@lamar.edu, anakano@usc.edu 


\section{Homology modeling}

Due to the flexibility of the N- and C-termini, the original crystal structure of Shewanella oneidensis cytochrome c MtrF (pdb code: 3pmq) misses certain residues around both terminals. We first established the homology modeling based on the original crystal structures of MtrF using the web server I-TASSER ${ }^{1}$, according to the sequences in the FASTA and our experimental insight on terminal residues as shown below. The italicized represent the newlyadded residues in the original crystal structures of MtrF in the protein data bank.

MtrF

CGGSDGDDGSPGEPGKPPAMTISSLNISVDKVAISDGIAQVDYQVSNQENQAVVGIPSATF IAAQLLPQGATGAGNSSEWQHFTSETCAASCPGTFVDHKNGHYSYRFSATFNGMNGVT FLSDATQRLVIKIGGDALADGTVLPITNQHYDWQSSGNMLAYTRNLVSIDTCNSCHSNL AFHGGRYNQVETCVTCHNSKKVSNAADIFPQMIHSKHLTGFPQSISNCQTCHADNPDLA DRQNWYRVPTMEACGACHTQINFPAGQGHPAQTDNSNCVACHNADWTANVHSNAAQ TSALAQFNASISSASMDANGTITVAVSLTNPTTGTAYADSADKLKFISDLRIYANWGTSF DYSSRSARSIRLPESTPIAGSNGTYSYNISGLTVPAGTESDRGGLAIQGRVCAKDSVLVDC STELAEVLVIKSSHSYFNMSALTTTGRREVISNAKCASCHGDQQLNIHGARNDLAGQCQ LCHNPNMLADATATNPSMTSFDFKQLIHGLHSSQFAGFEDLNYPGNIGNCAQCHINDST GISTVALPLNAAVQPLALNNGTFTSPIAAVCSNCHSSDATQNHMRQQGAVFAGTKADA TAGTETCAFCHGQGTVADVLKVHPINDDDDKAACCPGCCKGKPIPQPLLGLDSTRTGHH $\mathrm{HHHH}$ 


\section{Molecular dynamics simulation}

Full-atom molecular dynamics (MD) simulation was performed in the NVT ensemble at temperature 298.15 K. As shown in Fig. S1, the protein molecule (MtrF) with protonation states defined by Gromacs is solvated in water environment with $36 \mathrm{Na}^{+}$counter ions at $\mathrm{pH}$ 7.0. The $\mathrm{Au}$ (111) surface was built in the dimensions of $12.11 \times 11.99 \mathrm{~nm}^{2}$, containing 12096 gold atoms. The periodic boundary condition in $x$ - and $y$-directions was used in protein-adsorption MD simulation. Surface atoms were aligned with periodic boundary image atoms in accordance with the gold crystal lattice to mimic a large surface without boundary effects. Two repulsive walls were built on the top and bottom layers of the $z$-direction to confine solvent molecules. The temperature was maintained at $298.15 \mathrm{~K}$ via Nóse-Hoover thermostat and the velocity Verlet algorithm was applied with a time step of $1 \mathrm{fs}$. The particle mesh Ewald (PME) $)^{2}$ summation was adopted to calculate the long-range electrostatic interactions with a cut-off distance of $1.2 \mathrm{~nm}$, which is also the spherical cut-off distance for Lennard-Jones interaction. The long-range dispersion corrections for energy and pressure were also calibrated. The missing angle parameter of axial histidine $\mathrm{N}-\mathrm{Fe}-$ histidine $\mathrm{N}$ was represented by the existing histidine $\mathrm{N}-\mathrm{Fe}$-ligand $\mathrm{O}$ interaction. 


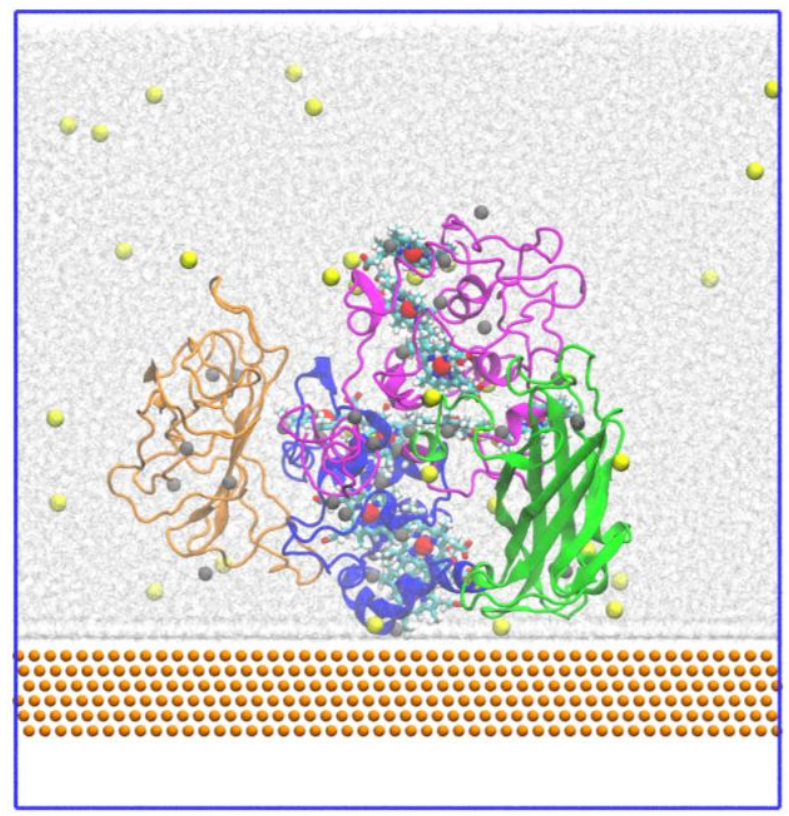

Figure S1. The initial configuration of MD simulation in water with counter ions $\left(\mathrm{Na}^{+}\right)$. The protein is colored orange, blue, green and purple, corresponding to domains I, II, III and IV. The sulfur atoms in the protein molecule are colored gray, the counter ions $\mathrm{Na}^{+}$yellow, the surface gold atoms orange, and water silver.

\section{Molecular mechanics/Poisson-Boltzmann surface area method}

To determine the orientation of the protein adsorbed onto the surface, the binding free energy between the gold surface and protein of different orientation was computed with molecular mechanics/Poisson-Boltzmann surface area (MM-PBSA) method ${ }^{3-8}$, which combines the Gromacs $^{9}$ software and the Adaptive Poisson-Boltzmann Solver (APBS) program ${ }^{8}$. The total free energy between the surface and the protein was estimated by taking into account the proteinsurface interactions and the dehydration/hydration energy for both surface and protein (Fig. S2). In MM/PBSA, the binding free energy was defined as the free energy difference of $\mathrm{A}$ and $\mathrm{B}$ in the aqueous environment as 


$$
\Delta G_{\text {binding }}=G_{a q u}^{A B}-G_{a q u}^{A}-G_{a q u}^{B}
$$

where $\Delta G_{\text {binding }}$ is calculated through the thermodynamic cycle in Fig. S2, including the complex solvation effects.

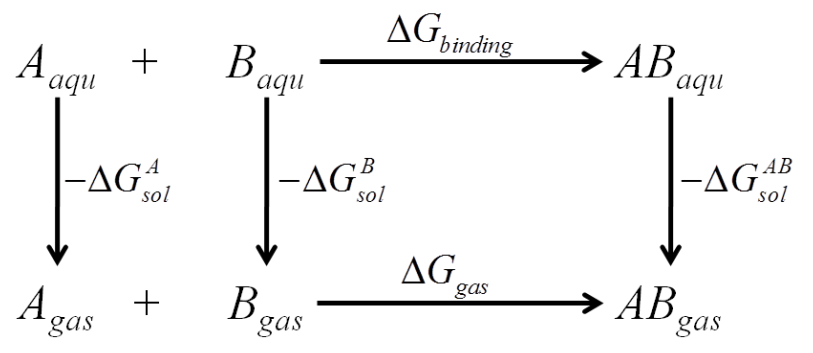

Figure S2. The thermodynamic cycle to calculate the binding free energy of two bodies, A and B.

The corresponding binding energy term was expressed as

$$
\begin{aligned}
& \Delta G_{\text {binding }}=\Delta G_{\text {gas }}-\Delta G_{\text {sol }}^{A}-\Delta G_{\text {sol }}^{B}+\Delta G_{\text {sol }}^{A B} \\
& \Delta G_{\text {gas }}=\left(\Delta\left\langle E_{\text {int } r a}\right\rangle+\Delta\left\langle E_{L J}\right\rangle+\Delta\left\langle E_{\text {coul }}\right\rangle\right)-T\left\langle\Delta S_{M M}\right\rangle \\
& \Delta G_{\text {sol }}^{i}=\Delta G_{\text {polar }}^{i}+\Delta G_{\text {nonpolar }}^{i}, i=A, B, A B \\
& \Delta G_{\text {polar }}^{i}=G_{\text {polar }, \varepsilon=80}^{i}-G_{\text {polar }, \varepsilon=1}^{i}, i=A, B, A B . \\
& \Delta G_{\text {nonpolar }}^{i}=\gamma_{i}\left\langle S A S A_{i}\right\rangle, i=A, B, A B . \\
& \Delta G_{\text {polar }}=\Delta G_{\text {polar }}^{A B}-\Delta G_{\text {polar }}^{A}-\Delta G_{\text {polar }}^{B} \\
& \Delta G_{\text {nonpolar }}=\Delta G_{\text {nonpolar }}^{A B}-\Delta G_{\text {nonpolar }}^{A}-\Delta G_{\text {nonpolar }}^{B}
\end{aligned}
$$

The binding free energy of each configuration includes the molecular interactions in the vacuum phase $\left(\Delta G_{g a s}\right)$ and the solvent effect contribution $\left(\Delta G_{\text {sol }}^{i}\right)$ in Equation 2, which corresponds to the thermodynamic cycle in Fig. S2. As shown in Equation 3, $\Delta G_{\text {gas }}$ consists of the contributions from the change of the intramolecular interactions $\left(E_{\text {intra }}\right)$, intermolecular interactions including Lennard-Jones $\left(E_{L J}\right)$ and Coulombic $\left(E_{c o u l}\right)$ and also the entropy change $\left(\Delta S_{M M}\right)$. We followed previous research works ${ }^{4-5,10}$ in ignoring the entropy contribution due to its inaccuracy and debatable contribution to the free energy. Intermolecular interactions were 
computed with molecular mechanics. Because protein was treated as a rigid body rotating in the Euler angle space, the change of the intramolecular force was also ignored. The solvation effects $\left(\Delta G_{s o l}^{i}\right)$ for component $(i=\mathrm{A}, \mathrm{B}$ and $\mathrm{AB})$ were reduced into the polar $\left(\Delta G_{p o l a r}^{i}\right)$ and nonpolar $\left(\Delta G_{\text {nonpolar }}^{i}\right)$ contributions as in Equation 4. The polar contributions $\left(\Delta G_{\text {polar }}^{i}\right)$ were calculated as the electron static energy difference of the protein-solvent system between the gas phase $(\varepsilon=1)$ and the solution phase $(\varepsilon=80)$ in a continuum implicit water environment (Equation 5). The nonpolar contributions $\left(\Delta G_{\text {nonpolar }}^{i}\right.$ ) were estimated from the solvent accessible surface area $(\mathrm{SASA})$ with the surface tension $\left(\gamma_{\text {gold }}=848.82 \mathrm{~kJ} \mathrm{~mol}^{-1} \mathrm{~nm}^{-2}, \gamma_{\text {protein }}=2.77 \mathrm{~kJ} \mathrm{~mol}^{-1} \mathrm{~nm}^{-2}\right)^{11-13}$ shown in Equation 6. A probe with the radius of $0.14 \mathrm{~nm}$ was used to identify the dielectric boundary for SASA estimation. $\Delta G_{\text {polar }}$ and $\Delta G_{\text {nonpolar }}$ shown in Equation 7 and 8 represent the itemized energy terms for the electrostatic and nonpolar contributions in the overall binding free energy. Table S1 lists the itemized energy for the most likely and unlikely configurations. Figure S3 shows the top-ranking candidates of four assemblies of energy-unfavorable configurations at the minimum protein-surface distance of $0.3 \mathrm{~nm}$.

Table S1. The itemized binding free energies of four most possible adsorption configurations and four least possible adsorption configurations.

\begin{tabular}{cccccc}
\hline$\theta$ (degree) & $\Psi$ (degree) & $\begin{array}{c}\Delta E_{\text {Coul\&LJ }} \\
(\mathrm{kT})\end{array}$ & $\Delta G_{\text {nonpolar }}(\mathrm{kT})$ & $\Delta G_{\text {polar }}(\mathrm{kT})$ & $\Delta G_{\text {binding }}(\mathrm{kT})$ \\
\hline 150 & 210 & -175.42 & -790.16 & 470.77 & -494.81 \\
\hline 60 & 300 & -113.39 & -555.78 & 266.06 & -403.11 \\
\hline 75 & 180 & -109.19 & -590.06 & 298.18 & -401.17 \\
\hline 45 & 225 & -95.53 & -489.75 & 246.76 & -335.52 \\
\hline 105 & 330 & -50.74 & -121.09 & 569.93 & 398.10 \\
\hline 105 & 345 & -33.01 & -101.47 & 542.14 & 407.65 \\
\hline
\end{tabular}




\begin{tabular}{llllll}
\hline 90 & 0 & -27.42 & -129.24 & 565.19 & 408.54 \\
\hline 90 & 15 & -48.10 & -140.99 & 643.74 & 454.65 \\
\hline
\end{tabular}

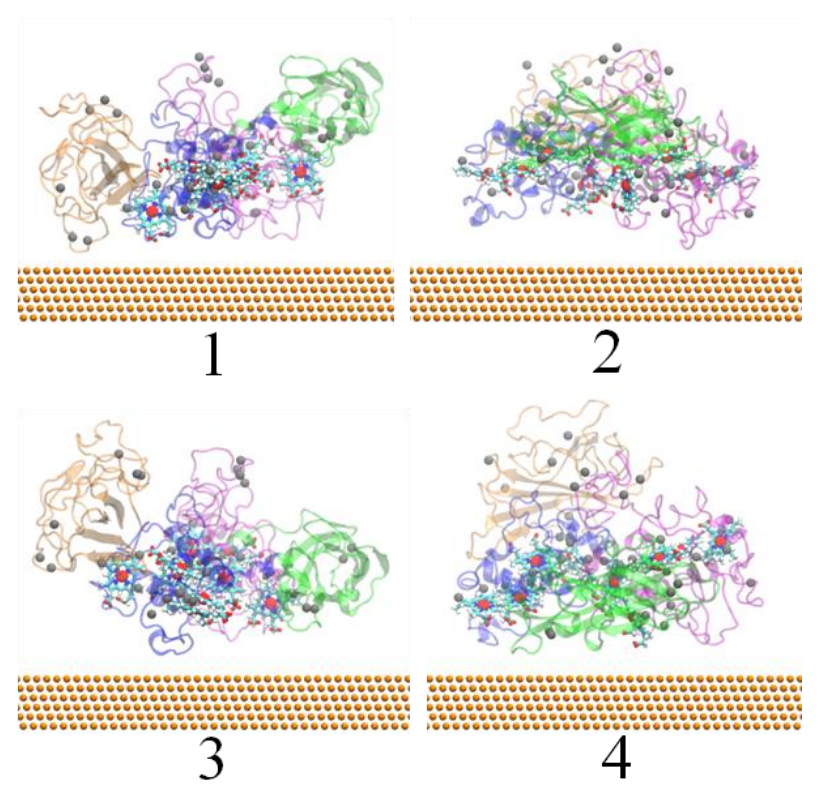

Figure S3. Four assemblies of the most energetically unfavorable configurations of the binding free energy $454.65 \mathrm{kT}, 408.54 \mathrm{kT}, 407.65 \mathrm{kT}$ and $398.10 \mathrm{kT}$, respectively. Iron ions are colored red, deca-hemes, cyan, and sulfur atoms grey.

\section{Kinetic Monte Carlo Simulations}

The kinetic Monte Carlo (KMC) simulation ${ }^{14-20}$ was performed to study the electron transfer (ET) dynamics. In each KMC step, one of the three events are chosen: (1) an electron injection into the network with rate $\alpha$ if the entrance heme is unoccupied; (2) an electron ejection from the network to the environment with rate $\beta$ if the exit heme is occupied; (3) an internal electron hopping from occupied heme $i$ to an unoccupied heme $j$ with rate $k_{j i}$. The probability of each particular event was proportional to its specific rate. An event was selected stochastically as 
follows: $L$ as the total number of the possible events and $k_{l}(l=1, \ldots, L)$ as the rate of the $l^{\text {th }}$ event. The specific event $l^{*}$ was chosen as

$$
\sum_{l=1}^{l^{*}-1} k_{l}<\xi_{1} k_{\text {total }}<\sum_{l=1}^{l^{*}} k_{l}
$$

where $\xi_{l}$ is a random number uniformly distributed in range $(0,1)$ and

$$
k_{\text {total }}=\sum_{l=1}^{L} k_{l}
$$

is the accumulative rate of all the possible events. The chosen event was performed and the time increment was defined as

$$
\tau=-\ln \left(\xi_{2}\right) / k_{\text {total }}
$$

where $\xi_{2}$ is another random number uniformly distributed in range $(0,1)$. KMC steps were repeated for $K\left(=10^{6}\right)$ times to describe the time evolutions until the system reached a steady state. Figure S4 shows the steady-state ET transfer across the heme-network of solvated MtrF in both directions (heme10-to-heme5 and heme5-to-heme10). The flux of heme10-to-heme5 is larger than that of the reverse direction. 

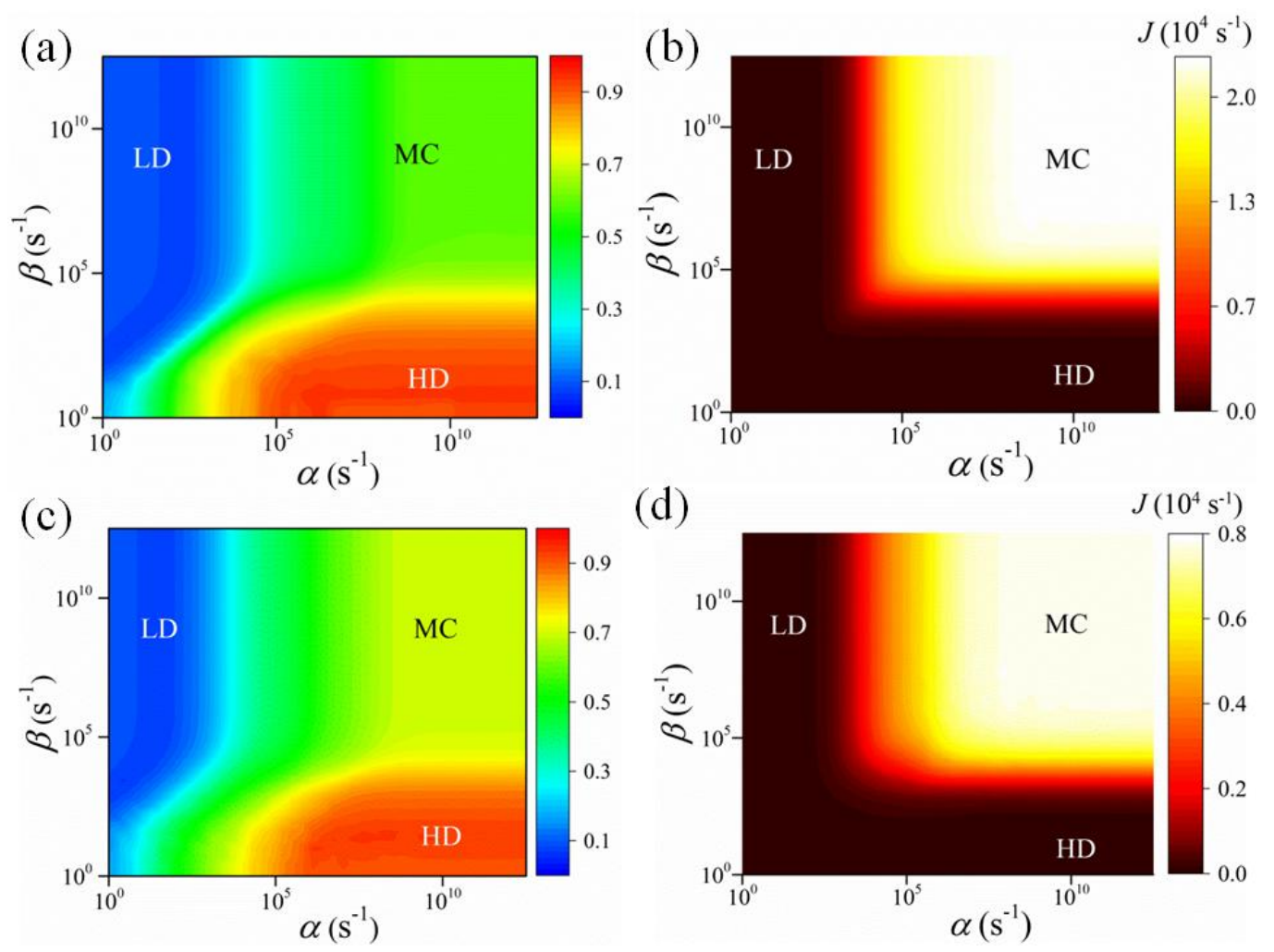

Figure S4. (a) and (c) Phase diagram of the time-averaged electron occupation density $\langle n\rangle$ for all 10 hemes as a function of the incoming $(\alpha)$ and outgoing $(\beta)$ ET rates for the solvated structure in the transfer direction of heme10-to-heme5 (from the environment to the surface through the protein) and heme5-to-heme10 (from the surface to the environment through the protein), respectively. (b) and (d) The corresponding phase diagrams of the net electron flux $J$ for the solvated structure in the transfer direction of heme10-to-heme5 and heme5-to-heme10, respectively.

Table S2. The maximum electron flux $\left(\mathrm{s}^{-1}\right)$ of different directions and adsorption status.

\begin{tabular}{|c|c|c|}
\hline Electron Flux $\left(\mathrm{s}^{-1}\right)$ & heme 10 to heme 5 & heme 5 to heme 10 \\
\hline Solvated Structure & $22845.72 \pm 2263.79$ & $7785.99 \pm 349.64$ \\
\hline Adsorbed Structure & $5905.92 \pm 2332.88$ & $4673.72 \pm 919.25$ \\
\hline
\end{tabular}




\section{Movies}

The movie file, movie1.avi, shows domain movements during 200-ns protein adsorption.

The movie file, movie2.avi, shows protein-surface thio-bonding during 200-ns protein adsorption.

\section{Reference}

1. Roy, A.; Kucukural, A.; Zhang, Y. I-TASSER: a unified platform for automated protein structure and function prediction. Nature Prot. 2010, 5 (4), 725-738.

2. $\quad$ Essmann, U.; Perera, L.; Berkowitz, M. L.; Darden, T.; Lee, H.; Pedersen, L. G. A Smooth Particle Mesh Ewald Method. J. Chem. Phys. 1995, 103 (19), 8577-8593.

3. Nakano, C. M.; Ma, H.; Wei, T. Study of lysozyme mobility and binding free energy during adsorption on a graphene surface. Appl. Phys. Lett. 2015, 106 (15).

4. Paissoni, C.; Spiliotopoulos, D.; Musco, G.; Spitaleri, A. GMXPBSA 2.0: A GROMACS tool to perform MM/PBSA and computational alanine scanning. Comput. Phys. Commun. 2014, 185 (11), 2920-2929.

5. Paissoni, C.; Spiliotopoulos, D.; Musco, G.; Spitaleri, A. GMXPBSA 2.1: A GROMACS tool to perform MM/PBSA and computational alanine scanning. Comput. Phys. Commun. 2015, 186, 105-107.

6. Massova, I.; Kollman, P. A. Computational alanine scanning to probe protein-protein interactions: A novel approach to evaluate binding free energies. J. Am. Chem. Soc. 1999, 121 (36), 8133-8143.

7. Srinivasan, J.; Cheatham, T. E.; Cieplak, P.; Kollman, P. A.; Case, D. A. Continuum solvent studies of the stability of DNA, RNA, and phosphoramidate - DNA helices. J. Am. Chem. Soc. 1998, 120 (37), 9401-9409.

8. Baker, N. A.; Sept, D.; Joseph, S.; Holst, M. J.; McCammon, J. A. Electrostatics of nanosystems: Application to microtubules and the ribosome. Proc. Natl. Acad. Sci. U. S. A. 2001, 98 (18), 10037-10041.

9. Hess, B.; Kutzner, C.; van der Spoel, D.; Lindahl, E. GROMACS 4: Algorithms for highly efficient, load-balanced, and scalable molecular simulation. J. Chem. Theory Comput. 2008, 4 (3), 435-447.

10. Hou, T.; Wang, J.; Li, Y.; Wang, W. Assessing the performance of the MM/PBSA and MM/GBSA methods. 1 . The accuracy of binding free energy calculations based on molecular dynamics simulations. J. Chem. Inf. Model. 2010, 51 (1), 69-82.

11. Heinz, H.; Vaia, R. A.; Farmer, B. L.; Naik, R. R. Accurate simulation of surfaces and interfaces of face-centered cubic metals using 12-6 and 9-6 Lennard-Jones potentials. J. Phys. Chem. C 2008, 112 (44), 17281-17290.

12. Sitkoff, D.; Sharp, K. A.; Honig, B. Correlating solvation free energies and surface tensions of hydrocarbon solutes. Biophys. Chem. 1994, 51 (2), 397-409.

13. Brown, S. P.; Muchmore, S. W. Large-scale application of high-throughput molecular mechanics with Poisson-Boltzmann surface area for routine physics-based scoring of proteinligand complexes. J. Med. Chem. 2009, 52 (10), 3159-3165. 
14. Byun, H. S.; Pirbadian, S.; Nakano, A.; Shi, L.; El-Naggar, M. Y. Kinetic Monte Carlo simulations and molecular conductance measurements of the bacterial decaheme Cytochrome MtrF. ChemElectroChem 2014, 1 (11), 1932-1939.

15. Nakano, C. M.; Byun, H. S.; Ma, H.; Wei, T.; El-Naggar, M. Y. A framework for stochastic simulations and visualization of biological electron-transfer dynamics. Comput. Phys. Commun. 2015, 193, 1-9.

16. Shimojo, F.; Hattori, S.; Kalia, R. K.; Kunaseth, M.; Mou, W.; Nakano, A.; Nomura, K.-i.; Ohmura, S.; Rajak, P.; Shimamura, K.; Vashishta, P. A divide-conquer-recombine algorithmic paradigm for large spatiotemporal quantum molecular dynamics simulations. J. Chem. Phys. 2014, 140 (18).

17. Bortz, A. B.; Kalos, M. H.; Lebowitz, J. L. A new algorithm for Monte Carlo simulation of Ising spin systems. J. Comput. Phys. 1975, 17 (1), 10-18.

18. Gillespie, D. T. A general method for numerically simulating the stochastic time evolution of coupled chemical reactions. J. Comput. Phys. 1976, 22 (4), 403-434.

19. Voter, A. F. Introduction to the kinetic Monte Carlo method. In Radiation Effects in Solids, Springer: 2007; pp 1-23.

20. Fichthorn, K. A.; Weinberg, W. H. Theoretical foundations of dynamical Monte Carlo simulations. J. Chem. Phys. 1991, 95 (2), 1090-1096. 